\title{
Schweizerische Bibliographie der Geschichte der Naturwissenschaften und der Medizin 1938-1943, IV. Serie
}

\author{
1. Geschichte der Naturwissenschaften: Allgemeines, Bibliographie
}

Binswanger, Ludwig. Karl Jaspers und die Psychiatrie. Schweiz. Arch. f. Neurol. u. Psychiat. 51, 1-13, (1943).

Boner, Georg. Geschichte des letzten Halbjahrhunderts der Freiwilligen akademischen Gesellschaft in Basel. Basel 1943.

Boner, Georg und Felix Stähelin. Die Universität Basel in den Jahren 1914 bis 1939. Basel, Verlag Fr. Reinhardt, 1943.

Goldschmidt, Günther. Von den medizinischen Handschriften zu Basel. Mitteilungen z. Gesch. d. Medizin, d. Naturwissenschaften u. d. Technik, 40, S. 359-361 (1941/42).

Nager, Rudolf F. Wesen und Unvollkommenheit der Naturwissenschaft unter besonderer Berücksichtigung der Arbeiten neuerer französischer Wissenschaftstheoretiker. Diss. Phil. Freiburg. 95 S. Freiburg (Schweiz). Paulusdr. 1938.

Niggli, Paul. Menschenbildung, Urteilskraft und Naturerkenntnis. Schweiz. Lehrerzeitung 88, 369-375 (1943).

Schlaginhaufen, Otto. Julius Klaus und die Julius Klaus-Stiftung für Vererbungsforschung, Sozialanthropologie und Rassenhygiene in Zürich. (2. Jahresber. der schweizer. Ges. f. Vererbungsforschg.) Arch. der Jul. Klaus-Stftg., 17, $462-464$ (1942).

Société de Physique et d'Histoire Naturelle de Genève. Cent-cinquantième Anniversaire de la Société. Genève 1940.

Stähelin, Felix siehe: Boner.

Uehlinger, Arthur: Zum 100jährigen Bestehen des Naturhistorischen Museums der Stadt Schaffhausen. Eröffnungsrede z. 123. Jahresversammlung der Schweiz. Naturforsch. Ges. vom 28.-30. Aug. 1943, in Schaffhausen. Verh. Schweiz. Naturf. Ges. 123. Sitzung, H. R. Sauerländer, Aarau.

\section{Geschichte der exakten Wissenschaften}

Dessauer, Friedrich. Der Fall Galilei und wir. Verlag Räber \& Co., Luzern, 1943.

Dhéré, Charles. Michel Tswett, le créateur de l'analyse chromatographique par adsorption. Sa vie, ses travaux sur les pigments chlorophylliens. (Avec fig.). Candollea, 10, 23-73 (1943).

Fischer, Hans. Zur Erinnerung an Antoine Laurent Lavoisier (1743-1794). Gesnerus 1, H. 2, p. 74-76 (1943).

Gonseth, F. Die Dialektisierung der Erkenntnis. E.T.H.-Schriften. Polygraphischer Verlag, Zürich 1943. 
Greiner, P. Isaak Newton, sein Leben und sein Lebenswerk. P. Haupt, Bern 1943. Naumann, W. Le Mathématicien et médecin bâlois Daniel Bernoulli (1700 bis 1782). Rev. Ciba, ann. 1943, p. 1029-1030.

3. Geschichte der Geologie, Geographie, des Alpinismus und der Technik

Grob, Richard. Geschichte der schweizerischen Kartographie. I. Teil, 98 S., 12 Tafeln. Jahresber. Geogr. Ges. Bern, Bd. 33 (1940). II. Teil, 85 S., 16 Tafeln. Jahresber. Geogr. Ges. Bern, Bd. 34 (1942).

Guyan, W. U. Die alten Eisenschmelzen von Merishausen. "Schaffhauser Mappe» (Neujahrsblatt auf 1943) Schaffhausen 1942.

Leicht, Hermann. Der Indianer-Bodmer. Neue Zürcher Zeitung 25. Dezember 1943.

Muller, Ed. Le rôle de la science dans l'industrie. Schweiz. Med. Wschr. 1940, $558-559$.

Speiser Felix. Geschichte des Museums für Völkerkunde in Basel. 1893 bis 1942. Verhandl. d. naturf. Ges. Basel 54, 265-280, Basel 1943.

Weisz, Leo. Unbekannte Verdienste Zürichs um die moderne Vermessungstechnik. Neue Zürcher Zeitung, Nr. 1995, 2060, Dez. 1943.

\section{Geschichte der biologischen Wissenschaften}

I. Anatomie, Zellenlehre, Entwicklungsgeschichte, Physiologie, Zoologie, Paläontologie

Asher, Leon. Der Anteil der Schweiz an der Entwicklung der Physiologie. Schweiz. Med. Wschr. 1938, 947-948.

Cushing, Harvey. Vesaliana. A Bio-Bibliography of Andreas Vesalius. XXXVIII $+230+86$ plates. Publication Nr.6, Historical Library, Yale University Library. New-York, Schumanns (1943).

Grünthal, E. Goethes Äußerungen zur Hirnanatomie. Monatsschr. f. Psychiatr. u. Neurol. 107 (1943).

Karcher, Hans. Andreas Vesalius de humani corporis Fabrica Libri VII. Verh. d. natf. Ges. Basel, Bd. 55, 1943/44. S. 194-208.

Ludwig, Eugen. Andreas Vesalius. Ansprache zur Feier des 400. Jahrestages des Erscheinens seiner «Fabrica corporis humanis» 1543. Verh. d. natf. Ges. Basel, Bd. 55, 1943/44. S. 209-210

Rudolf, Friedrich. Ein Erinnerungsblatt an Andreas Vesalius (1 Taf.). Basler Jahrb., 1943, 113-121.

Schwerz, Franz. Zur 300. Jahresfeier von John Mayow, dem Entdecker des Wesens der Verbrennungs- und Atmungschemie. Praxis, 32, 330-331, 1943.

Straub, Hans. Die Werke Vesals. Verh. d. natf. Ges. Basel, Bd. 55, 1943/44, S. $235-240$. 
Thurnherr, Margrit. Benennungsmotive bei Insekten untersucht an schweizerdeutschen Insektennamen, unter besonderer Berücksichtigung der Ostschweiz. Diss. Phil. I. Univ. Zürich (mit Abb.) XVI + $191 \mathrm{~S}$. Winterthur, Buchdr. Winterthur, 1938.

Verzár, Fritz. Andreas Vesalius in Basel. Zur Feier des 400. Jahrestages des Erscheinens seiner "Fabrica corporis humanis» 1543. Verh. d. natf. Ges. Basel, Bd. 55, 1943/44, S. 180-193.

Wolf-Heidegger, Gerhard. Vesals Basler Skeletpräparat aus dem Jahre 1543. (Mit 1 Tafel und 1 Textfigur.) Verh. d. natf. Ges. Basel, Bd. 55, 211-234. 1943/44).

\section{Geschichte der Botanik; Forstwirtschaft, Landwirtschaft}

Dolf, Willy. Die ökonomische-patriotische Bewegung in Bünden. Ein Beitrag zur bündnerischen Wirtschafts- und Geistesgeschichte des 18. Jahrhunderts. H. R. Sauerländer, Aarau, 1943.

Forrer, Jacob. Geschichte und Aufbau der Braunviehzucht des Kantons Graubünden. Diss. Vet.-Med. Univ. Zürich (Mit Tabellen und 3 Tafeln). 88 S. Chur, Buchdr. Bischofberger \& Co., 1938.

Stiefel-Bianca, Anita. Das Wirken der ökonomischen Kommission in der zürcherischen Landschaft. Diss. Phil. I. Zürich 1943.

\section{Geschichte der Medizin}

einschließlich Zahnheilkunde, Veterinärmedizin, Pharmazie

Alfieri, E. Una traduzione italiana finora sconosciuta del «Rosengarten» di Eucharius Roesslin (Eucario Rhodiones). Schweiz. Med. Wschr. 1941, 1233.

Bernoulli, René. Geschichte der Soldatenernähıung der militärisch wichtigsten Völker Europas. Diss. med. Basel 1943. Allg. schweizer. Militärzeitung 89, 50 S. (1943).

Bertrand, J. B. Notes sur la santé publique et la médecine en Valais jusqu'au milieu du XIX ${ }^{e}$ siècle. Annales Valaisannes, Bulletin de la Société d'histoire du Valais romand. 60 S. illustr. Saint-Maurice 1940.

Bueß Heinrich. Andreas Ruinella (ca. 1555 bis 1620?), ein wenig bekannter Bündner Humanist, über die Zurückhaltung der Menses. Gesnerus 1, H.2, p. 37-58 (1943).

- Z Zeittafel zur Geschichte der Embryologie. Ciba Zeitschr. Jg. 8, S. 3228 (1943).

Denzler, Alice. Medizingeschichtliches aus der ersten Hälfte des 19. Jahrhunderts. (Aus den Berichten der Thurgauischen Bezirksärzte 1839-1862). Schweiz. Med. Wschr. 1938, 1300, 1320, 1381. 
Dreyfus, Camille. A propos des pilules de Méglin. Schweiz. Med. Wschr. 1940, 1228.

- - Montesquieu et la médecine. Schweiz. Med. Wschr. 1942, 497.

v. Fellenberg, R. Behandlung des Skorbutes im Jahre 1806. Schweiz. Med. Wschr. $1939,1311$.

Fischer, Hans. Medizinische Gelehrsamkeit in früheren Jahrhunderten. «Heilende Schweiz». S. 27-33, Schweiz. Zentrale für Verkehrsförderung. Zürich 1941.

- - Schweizerische Bibliographie der Geschichte der Naturwissenschaften und der Medizin. 1938-1943. I. Serie. Gesnerus 1, H. 2, p. 64-74 (1943).

Fringeli, Albin. Jakob Gerni, ein Landarzt vor 100 Jahren. (Mit Portr.). Dr Schwarzbueb, Soloth. Jahr- und Heimatbuch, 21, 91-95, (1943).

Fritzsche, Ernst. Schweizerische Gesellschaft für Chirurgie. Die ersten 25 Jahre ihres Bestehens 1913-1938. Schweiz. Med. Wschr. 1938, 578-587.

Galewski, Salomon. Versuch einer pragmatischen Geschichte der manuellen Hilfe bei der Geburt. Thèse méd. Lausanne, 62 p. Lausanne, Impr. C. Risold \& Fils, 1937-1938.

Gallardo-Diaz, José A. La médecine au mexique pendant la période précoloniale et coloniale. Thèse Méd. Lausanne, 28 p. Lausanne, Impr. C. Risold \& Fils, 1938.

Gigon, Alfred. Die bedeutendsten Schweizer Ärzte des 19. und 20. Jahrhunderts. «Heilende Schweiz». S. 39-41, Schweiz. Zentrale für Verkehrsförderung, Zürich 1941.

Goldschmidt, Günther. Ein Pseudo-Apuleiusfragment in einer Zürcher Handschrift. Gesnerus 1, H. 2, p. 59-63 (1943).

Gordonoff, Theodor. Der Anteil der Schweiz an der Entwicklung der Medizin. In: «Die Schweiz und die Forschung;... h. h. v. Walter Staub und Adolf Hinderberger. Bern u. Freiburg, Verl. des Guide pratique, 1943, S. 18-29.

Grange, Marguerite. La Croix-Rouge, son histoire et son œuvre. (Avec des fig.). Neuchâtel, Paris, Delachaux \& Niestlé S. A., (1943), IV + 24 p. Cahiers d'enseignement pratique. No. 35 .

Greil, Alfred. Entwicklung und Zukunft der Schulmedizin. Schweiz. Med. Wschr. 1940, 281.

Guisan, André. Historique de l'opération césarienne. Rev. Vesk̄a, 7, 15-17, (1943).

- La saignée dans le passé. Rev. Veska, 7, 52-54 (1943).

- - Un service de chirurgie à la fin du XVIIIe siècle. Rev. Veska, 7, 101102 (1943).

Häni, A. Ein hervorragender Arzt vor $100 \mathrm{Jahren}$ (Dr. Heußer, Hombrechtikon). Zürichsee-Zeitung 27. April 1944, Nr. 98. 
Hauduroy, Paul. Alexandre Yersin et la découverte du bacille pesteux. Notice nécrologique. Schweiz. med. Wschr. 1943, 750-751.

Henschen, C. Über das Keratoderma senile volae manuum et plantae pedum. (Medizin-historischer Nachtrag zu der Arbeit Schweiz. Med. Wschr. Nr. 29, 1940). Schweiz. Med. Wschr. 1941, 97-98.

Hopf, Max. 25 Jahre Schweizerische Röntgen-Gesellschaft. Schweiz. Med. Wschr. 1938, 529-533.

Hüssy, Paul. Krankheit und Tod bedeutender Menschen. Veska-Zeitschr. 7, 95-100 (1943).

Jenny, Ed. Zum 100. Geburtstag von Gotthelfs Anne Bäbi Jowäger. Vortrag, gehalten an der Jahresversammlung der Schweiz. Gesellschaft für Pädiatrie, am 3. Juni 1944 in St. Gallen. Schweiz. Med. Wschr. 1945, 68.

Jenny J. J. Unhygienische Moden. (Mit Abbildungen). Ciba Zeitschr. 8, 31143152, (1943).

Jojeux, Ch. Le paludisme, extension et regression. Bull. Soc. Neuchâteloise des Sciences Naturelles 67, 5-97 (1942).

Jung, P. Zur Geschichte der Sectio in mortua in der Schweiz. Schweiz. Med. Wschr. 1941, 1287-1288.

Karcher, Hans. Vermag Sydenham dem modernen Arzte noch etwas zu sagen? Schweiz. med. Wschr. 1940, 233-239.

- - Über einige berühmte schweizerische Praktiker aus der Zeit der Aufklärung. Schweiz. Med. Wschr. 1941, 887, 914, 938.

Keller, Robert. L'hermaphrodisme. (Avc fig.). Rev. Ciba, ann. 1943, p. 870-894.

Kertész, Robert. Ignaz Semmelweis, der Kämpfer für das Leben der Mütter. Rascher \& Co. Zürich 1943.

Kipnis, Walter Seth. Beitrag zum Studium des Ajurveda, des Heilsystems der Inder. Diss. Méd. Lausanne, Impr. C. Risold \& Fils, 1938.

Koch, Hans. Franz Karl Stadlin, 1777-1829. Zuger Köpfe. 50 Lebensbilder aus dem Zugerlande. Zug, Eberhard Kalt-Zehnder 1943, S. 183-188.

Kocher, Paul E. Zum Zentenarium der Schmerzbetäubung. Schweizer. Monatsschr. f. Zahnheilk. 53, 1105-1110, (1943).

Lehmann, Heinrich. Krätzeheilmittel und Krätzekuren im Wandel der Zeiten. Pharm. acta Helv. 18, 203-214, (1943).

Linder, David Osias. Die Geschichte der Buergerschen Krankheit. Diss. Méd. Lausanne, 24 S. Lausanne, Impr. C. Risold \& Fils, 1938.

Makar, Aziz. Contribution à l'étude de l'épidémiologie de la peste. L'histoire et l'évolution de la peste en Egypte. Thèse Méd. Lausanne (Avec des graphiques et 1 croquis de carte) 52 p. Lausanne, Impr. C. Risold Fils, 1938.

Mauderli, Otto. Beitrag zur Geschichte der Wohnungshygiene der Stadt Basel. Zürich und Leipzig, Gebr. Leemann \& Co. 1943, 83 S. Zürcher medizingesch. Abh. 19, (Erschien auch als Basler Diss.). 
Milt, Bernhard. Zur Geschichte des schweizerischen Irrenwesens im 17. Jahrhundert. Schweiz. Med. Wschr. 1938, 1061-1062.

Misirlioglu, Jusuf Izzettin. Entwicklung und heutiger Stand der pathogenetischen Anschauungen über Apoplexia sanguinea. Diss. med. Basel 1943. Ausg. A.: II + 56 Bl. In Maschinenschrift. Ausg. B: Teildruck, 12 S.

v. Muralt, Alexander. Johannes v. Muralt, Stadt-Arzt zu Zürich, 1645-1733. Schweiz. med. Wschr. 1938, 511-513.

Naumann, W. Le mathématicien et médecin bâlois Daniel Bernoulli (17001782). Rev. Ciba, ann. 1943, p. 1029-1030.

Neumann, Heinrich. Die medizinische Philosophie. Alte und neue Forschungsmethoden. Der moderne Gelehrte. Die Überwucherung der Spezialisierung. Zweck der ärztlichen Wissenschaft. Die Ärzteausbildung. Ärztliche Ethik und Weltanschauung. Systematische Organisierung. Aufgaben der medizinischen Philosophie. Schweiz. Med. Wschr. 1940, 819-821.

Olivier, E. Menues notes sur le passé médical du Pays de Vaud. 16. Notes sur la maladière de Colovray près Nyon. Rev. historique vaudoise 51, 161 (1943).

Olivier, Jean. Aperçu de l'histoire de la médecine à Genève. Glandes endocrines et vitamines. Genève, Les Presses académiques, 1943, p. 711-724.

Podach, E. F. Historisches zur Jodmangeltheorie des Kropfes. Schweiz. Med. Wschr. 1940, 794.

de Quervain, F. Appendicitis vor 50 Jahren. Schweiz. Med. Wschr. 1939, 128129.

Reitzer, Hermann. Die Organisation des Ärztestandes in Griechenland. Schweiz. Med. Wschr. 1940, 401.

Renner, Eduard. Über das Magische und Animistische im Erleben und Denken der Urner Bergbauern. Diss. Med. Bern. I $+174+$ Zusammenfassung $5 \mathrm{Bl}$. Bern 1937 (in Maschinenschrift).

Robert, P. Zur Bilanz der modernen klinischen Medizin. Schweiz. Med. Wschr. 1943, 338.

Roch, Maurice. Réflexions sur une vieille thèse traitant de l'action analgésique des injections d'eau distillée. Schweiz. Med. Wschr. 1943, 1174-1175.

Roos, Adolf. Orthopädie vor 200 Jahren. (Mit Abbildungen.) Schweiz. Med. Wschr. 1938, 350-354.

Rufer, Alfred. Schiferlis Bericht aus dem Jahre 1802 an den helvetischen Senat über die Organisation des Medizinalwesens der helvetischen Truppen. Schweiz. Med. Wschr. 1939, 1311-1314.

Schiller, Dr. Die Geschichte von der Gründung und Entwicklung des Ärztevereins des Kantons St. Gallen seit 75 Jahren. (Vortrag an der Herbstversammlung 7. Oktober in Wattwil.) Schweiz. Med. Wschr. 1938, 27-29. 
Schmid, Alfred. Albrecht von Haller und seine Bedeutung für die Heilkunde. «Heilende Schweiz». S. 37-38, Schweiz. Zentrale für Verkehrsförderung, Zürich 1941.

- - Der Anteil der Schweiz an der medizinisch-literarischen Tätigkeit (vom Ende des 15. bis zum Ende des 18. Jahrhunderts). In: "Die Schweiz und die Forschung;... h. h. v. Walter Staub und Adolf Hinderberger. - Bern und Freiburg, Verl. des Guide pratique, 1943, S. 243-257.

Schwerz, Franz. Medizinstudenten im Kampf mit der Regierung um bessere Berücksichtigung einheimischer Dozenten an der Universität Zürich im Jahre 1859. Praxis, 32, 677-678, (1943).

Staehelin, J.E. Zur Geschichte der Lehre von den Temperamenten. Schweiz. Med. Wschr. 1941, 1401-1402.

Steiner, Peter. Das Chirurgenwesen Basels im Anfang des 19. Jahrhunderts. Schweiz. Med. Wschr. 1938, 1234-1236,

Steinmann, Alfred. Masken und Krankheit. (Mit Abb.) Ciba Zeitschr. 8, 3114$3152,(1943)$.

Tecoz, Henri-François. Les étapes historiques de la transfusion su sang. Praxis, 32, 382, 399, (1943).

Waller, Erik. Arnold C. Klebs, 1870-1943. Lychnos 1943, 439-441, Uppsala und Stockholm 1943 (Schwedisch).

Walther, Erwin. Zur Geschichte des Schularztwesens der Stadt Basel mit besonderer Berücksichtigung des schulärztlichen Dienstes. Diss. Med.-Dent. Basel. (Mit 2 Tabellen) 73 S. Buchdr. J. Heuberger, Basel 1937.

Wegelin, C. Pathologisch-anatomische Demonstrationen. 6. Sitzung vom 28. Januar 1943 des Medizinischen Bezirksverein Bern-Stadt. Schweiz. Med. Wschr. 73, 1415 (1943).

Wieland, Emil. Die Entwicklung der Pädiatrie in der Schweiz. Schweiz. Med. Wschr. 1939, 723-727.

Wilder, Francis D. Zur Geschichte des Kampfes gegen das Gelbe Fieber bei der Erbauung des Panamakanals. Diss. Med. Lausanne 1938, 31 S. Lausanne, Impr. C. Risold \& Fils.

Witz, Werner. Über die Cholera asiatica im Kanton Aargau anno 1854 und die dagegen ergriffenen Maßnahmen und Erlasse (5 Tab.). Diss. med.-dent. Basel. Zürcher med. gesch. Abh., 20, 49 S. (1943).

Wüscher, H. Haben die alten Autoren für uns überhaupt einen Wert? Schweiz. Med. Wschr. 1939, 706-707.

Zehnder, Alfred. Die Verköstigung der Kranken in den Basler Spitälern vom 15. bis 19. Jahrhundert. Diss. Med.-dent. Basel, 37 S. Buchdr. H. Studer-Meyer, 1938. 
Zwahlen, Georges. Contribution à l'histoire des couronnes et appareils à pont en Suisse (8 pl.). Thèse méd. dent. Genève. - Rev. mens. suisse d'odontol., 53, 40-75, 146-168 (1943).

\section{Nachtrag zu:}

Paracelsus-Literatur 1938-1943 (Gesnerus Jahrgang 2, Heft 1, S. 34, 1945)

Achelis, J. D. Über die Syphilisschriften Theophrasts von Hohenheim. I. Die Pathologie der Syphilis. Mit einem Anhang: Zur Frage der Echtheit des dritten Buches der Großen Wundarznei. Sitz. ber. Heidelberger Akad. Wiss., Math.-naturw. Kl., Jahrg. 1938, 9. Abhandlung.

- - Die Überwindung der Alchemie in der paracelsischen Medizin. Sitz. ber. Heidelberger Akad. Wiss., Math.-naturw. Kl., Jahrg. 1942, 3. Abhandlung.

Atzrott, E. H. G. Bombastus Philippus Aureolus Theophrastus von Hohenheim, gen. Paracelsus. Ein mittelalterliches Forscher- und Wanderleben. Hippokrates 7, 388-393 (1936).

Darmstädter, Ernst. Paracelsus. Seine Bedeutung für unsere Zeit. Natur und Kultur (München) 30, 442-444 (1933).

Diepgen, Paul. Paracelsus und seine Stellung in der historischen Entwicklung der Medizin. Klin. Wschr. 1942, 935.

Elze, C. Theophrastus Paracelsus. Klin. Wschr. 1942, 239.

—- Des Paracelsus Versuch der Medizinreform. Klin. Wschr. 1942, 591/593.

Englert, Ludwig. Mensch, Arznei und Arzneibereitung bei Paracelsus. D. Pharmzeut. Industrie, 1935, H. 3.

Haering, Theodor. Cusanus - Paracelsus - Böhme. Ein Beitrag zur geistigen Ahnenforschung unserer Tage. Z. f. deutsche Kulturphilosophie 2, 1-25 (1935).

Haering, Theodor und E. Metzke. Erfahrung und Natur in der Gedankenwelt des Paracelsus. Blätter f. deutsche Philosophie 13, 193 (1939).

Heimsoeth, H. Paracelsus, Arzt, Philosoph. (Festvortrag anläßlich der Paracelsus-Feier der Arbeitsgemeinschaft deutscher Ärzte in Prag, Sitz. 28. Nov. 1941.) Klin. Wschr. 1942, 262.

Hooykaas, Reijer. Die Elementenlehre des Paracelsus. Janus (Leyden) 39, 175-187 (1935).

Hult, O. T. Om Paracelsus i äldre och nyare forskning. Lychnos, 1, 182-207 (1936).

Keller, A. Paracelsus: Von der krafft, würcklung und eigenschafft der Salzsohlen. Rheinfelden 1944.

Koyré, A. Paracelse. Revue d'hist. et de philos. religieuses, 13, 46-75; 145-163 (1933). 
Marx Helmut. Die Gestalt des Paracelsus. Klin. Wschr. 20, 935-939, 958-961 (1941).

Milt, Bernhard. Paracelsus. 1493-1541. «Heilende Schweiz». S. 36-37 (1941) Schweiz. Zentrale für Verkehrsförderung Zürich 1941.

Paracelsus. Theophrastus v. Hohenheim, gen. Paracelsus. Sämtl. Werke in zeitgemäßer kurzer Auswahl. Bd. I.: P. der Medizin- und Kulturhistoriker. Herausg. v. Dr. Jos. Strebel, Luzern. Verlag Zollikofer, St. Gallen, 1944.

Seckendorf, Ernst. Ein Urteil (Georg Palms) über Paracelsus in Randbemerkungen aus dem 16. Jahrhundert. Medizin. Mitteilungen 6, 51-56 (1934).

- - Darf man Paracelsus den «medizinischen Luther» nennen? Med. Welt 9, $433-435$ (1935).

Strunz, Franz. Theophrastus Paracelsus. Scientia 54, 149-158 (1933).

- - Theophrastus Paracelsus in der Geschichte der Chemie. (Vortrag.) Österr. Chem. Ztg. (N. F.) 37, 12-14 (1934).

- - Theophrast von Hohenheim genannt Paracelsus. "Von deutscher Art in Sprache und Dichtung», Bd. III., Stuttgart (1941), S. 97-146.

Stickel, Dietrich, Stiegele, Koch. Der deutsche Arzt. Paracelsus-Feier der Universität Tübingen. 44 S. Tübingen: Verl. v. J. C. B. Mohr (Paul Siebecke) 1941.

Sudhoff, Karl. Paracelsus-Bildnisse. Eine Aufklärung. Münch. med. Wschr. 1936, Nr. 2, p. 62. (S.-A., 7 pp.).

Titley, Arthur, F. Paracelsus, A Résumé of some Controversies. Ambix, Vol. I, No. 3, 1938, 166-183.

Weinhandl, Ferd. Paracelsus. "Das Deutsche in der deutschen Philosophie», herausg. v. Theod. Haering, Verlag G. Kolhammer, Stuttgart 1941, p. 89-139.

Hans Fischer. 